<smiles>[C]1C=C[CH-]C1</smiles>

\title{
La evaluación educativa siempre a prueba
}

Educational assessment always on the test

Avaliação educacional sempre em julgamento

\author{
Ricardo Gutiérrez-Barba \\ Universidad de Guadalajara (México) \\ https://orcid.org/0000-0002-6752-8411
}

D0l: https://doi.org/10.35622/j.rep.2021.03.006

Recibido 03/09/2020/ Aceptado 20/08/2021

RESUMEN. La naturaleza y concepto de la evaluación educativa en la actualidad queda muy distante de su aplicación en la práctica. Se puede entonces hablar de dos realidades muy dispares: una la teorizante y por otro lado la puesta en práctica de este proceso tan fundamental en todos los sistemas educativos. En el presente escrito se analiza parte de la literatura que al tema se refiere, concentrando la atención en la realidad latinoamericana actual. La implementación de la evaluación educativa aún es reciente en nuestro contexto, ello supone que existen escollos y retos sustanciales que no se pueden postergar, entre ellos se pone especial énfasis en el renglón de la comunicación y la información de las acciones, resultados e implicaciones de este proceso evaluatorio.

PALABRAS CLAVE: Evaluación educativa, comunicación, información, proceso, retos.

ABSTRACT. The nature and concept of educational evaluation today are very far from its application in practice. One can then speak of two very disparate realities: one theorizing and, on the other hand, the implementation of this fundamental process in all educational systems. In this writing, part of the literature on the subject is analyzed, concentrating attention on the current Latin American reality. The implementation of educational evaluation is still recent in our context; this implies that some substantial obstacles and challenges cannot be postponed; among them, particular emphasis is placed on the line of communication and information on the actions, results, and implications of this evaluative process.

KEYWORDS: Educational evaluation, communication, information, process, challenges.

RESUMO. A natureza e o conceito de avaliação educacional hoje estão muito longe de sua aplicação na prática. Pode-se então falar de duas realidades muito díspares: uma teorizando e, por outro lado, a implementação desse processo fundamental em todos os sistemas educacionais. Nesta redação, analisa-se parte da literatura sobre 0 assunto, concentrando a atenção na realidade latino-americana atual. A implementação da avaliação educacional ainda é recente em nosso contexto, o que implica que existem obstáculos e desafios substanciais que não podem ser adiados, entre eles destaque especial para a linha de comunicação e informação sobre as ações, resultados e implicações deste processo avaliativo.

PALABRAS CLAVE: Avaliação educacional, comunicação, informação, processo, desafios. 


\section{INTRODUCCIÓN}

\section{Entre definiciones y algunas ambigüedades}

El tema de la evaluación educativa está indisolublemente unido al tema de la calidad en la educación, razón por la cual en la literatura científica se vuelve necesario establecer linderos en los conceptos para evitar ambigüedades y no perder objetividad en el análisis. A simple vista, el concepto de calidad es un concepto claro, contundente, suficientemente estudiado y ampliamente utilizado como para caer en la indefinición. Viene de la zona de las ciencias administrativas como filosofía de producción y herramienta de procesos tecnológicos, por tal motivo, no puede carecer de definición. El problema estriba en el momento en que se toma prestado el concepto y se siembra en la parcela de la educación.

Iniciemos con una analogía, estableciendo que, en la fábrica, en la empresa el producto final es pesado, medido, examinado, es decir, calificado para la venta al público, estamos ante un proceso de calidad y evaluación de ese proceso. Sin embargo, en educación no podemos estandarizar de esa forma, puesto que hay variables tan diversas que no pueden enfrascarse en un molde preestablecido. Recordemos que el concepto de calidad tuvo su mayor auge en tiempos de la postguerra y que fue en el ámbito empresarial donde nace como prevención de errores en la producción. Surge entonces la interrogante ¿acaso el sistema educativo necesite crear su propio sistema de calidad?

Es en este contexto, en las sociedades post industriales donde la rentabilidad de los procesos educativos tiene su origen, marcada con un fuerte interés en evaluar, la educación en un primer momento toma un perfil cuantitativo ya que considera el acceso de la población a la educación (Fernández, 2008). Es decir, el sistema educativo es exitoso siempre que la mayor cantidad de ciudadanos ingresen al mismo. De igual manera, se crean organismos que den respuestas objetivas y fiables, como son la demanda de información y resultados cuantitativos para confrontar al Estado, en caso de que éste sea un mal administrador.

Por otro lado, se ha de tomar en cuenta que en la educación los resultados son muy diversos y amplios. Es necesario considerar que el sistema educativo está integrado por subsistemas interdependientes y cada uno de ellos bien diferenciado y además con grados de 
complejidad cambiantes. Estos elementos del sistema educativo lo integran los alumnos, docentes, directivos, instituciones, programas y políticas públicas, en todos ellos hay insumos, procesos y resultados que, si no se toman en cuenta de manera integral, toda pretensión de evaluación quedará en números ilustrativos de una irrealidad. Por este motivo es necesario recordar que en educación la cantidad no implica mejoría. Hacer hincapié en el hecho evidente de que proceso y producto son elementos de una misma realidad no siempre bien analizada.

\section{DESARROLLO}

Por definición se entiende a la evaluación educativa como un fenómeno habitualmente circunscrito al aula, referido a los alumnos y limitado al control de los conocimientos adquiridos a través de pruebas de diversos tipos. Sin embargo, alrededor de esta actividad, existen cuestiones sobre la patología de la evaluación educativa, haciendo palpables los problemas de las limitaciones, las desviaciones y las manipulaciones de las que puede ser objeto (Santos, 2002). En no pocas ocasiones se plantea de forma negativa, puesto que es realizada en malas condiciones y utilizada de forma poco arbitraria. Es implementada de forma jerárquica y por lo tanto permite saber pocas cosas de cómo se produce el aprendizaje. Pocas veces sirve para mejorar la práctica de los profesores y desde luego, el contexto y el funcionamiento de las escuelas.

Es necesario recalcar que la evaluación educativa es una parte integrante de los proyectos, no algo añadido al final de los mismos, como un complemento o un adorno que se pondrá en funcionamiento si queda tiempo y si se tienen a bien. Si se diseña, planifica y pone en funcionamiento será imprescindible conocer qué es lo que se consigue por el hecho mismo de poner la iniciativa en acción, por qué esa y no otra, por qué de ese modo y para esos fines (Ruíz, 1996).

En este sentido, la evaluación educativa propiciará una mejora continua en los procesos y ámbitos en donde se intente aplicar de manera clara y pertinente, lo que conducirá a crear un ecosistema de comprensión, tolerancia y diálogo (Casanova, 2002). En relación a los programas, habrá una incidencia en la revisión de que lo que esté funcionando sea bueno. De acuerdo con Martínez (2016) la evaluación educativa constituye, en la actualidad, una 
actividad relativamente consolidada en Latinoamérica. Más de tres décadas han transcurrido ya desde las primeras iniciativas, muy incipientes, que buscaban conocer de manera sistemática procesos y resultados del sistema educativo. En la última década se han producido avances muy importantes, los cuales han modificado nuestra imagen del sistema educativo y sobre cómo debe evaluarse.

Hoy, si bien con diferencias en cuanto a su concepción, la mayor parte de los actores educativos coincide en la importancia de realizar evaluaciones periódicas de diferentes aspectos del sistema educativo (Jiménez, 2002). Resulta difícil imaginar la posibilidad de reflexionar o intervenir sobre la educación nacional sin tener en cuenta esta herramienta. En un período relativamente breve nos hemos habituado a la existencia de múltiples evaluaciones, cuyos resultados son públicamente difundidos y concitan, aunque brevemente, la atención del conjunto de la sociedad (Zorrilla, 2002). La mayor parte de estas evaluaciones se realiza de acuerdo a estándares conceptuales y metodológicos aceptados por la mayoría de los actores involucrados. Sin embargo, el camino de la evaluación aún enfrenta desafíos.

Por una parte, se asiste a una expansión acelerada de distintas iniciativas de evaluación que podría superar la capacidad del sistema educativo para asimilar y aprovechar sus resultados. Del otro lado, este proceso aún está incompleto. El vínculo entre datos y decisiones en los distintos niveles del sistema es aún débil, y las evaluaciones no impactan de manera decisiva en las políticas y programas. La distribución de atribuciones entre los organismos de evaluación no está claramente definida, y éstos aún no cuentan con la autonomía suficiente para garantizar su independencia conceptual y técnica. En muchas localidades latinoamericanas no se han desarrollado aún las capacidades para producir e interpretar información de manera rigurosa. Muchos actores en la base del sistema, principalmente maestros y directores, mantienen una fuerte resistencia a las evaluaciones estandarizadas (Martínez y Blanco, 2010).

En los últimos tiempos se habla mucho de evaluación educativa, sin hacer distinción de conceptos y finalidades, por tal motivo, se puede caer en imprecisiones al momento de referirnos a este proceso (Jiménez, 2002). Se habla de evaluación indicando al proceso de 
carácter comparativo aplicado a mediciones de resultados. Se llama evaluación a fenómenos de rendimiento de cuentas impuestas por la autoridad, se denomina evaluación al análisis diagnóstico realizado mediante instrumentos que permiten cuantificar los datos, se habla de evaluación cuando se realiza la comprobación del aprendizaje de los alumnos (Lafourcade, 2002). Sin embargo, la evaluación es mucho más que una tasación o de una comparación de un proceso de rendimiento de cuentas, se debe entender a la evaluación educativa como un proceso de indagación sobre el valor educativo de un programa, de su importancia, exigencias y significados ulteriores. Para evaluar hay que comprender, según la premisa de Stenhouse (citado por Medina 2013).

No debemos olvidar que lo más importante no es el hecho de realizar la evaluación, ni siquiera el modo de hacerla, sino al servicio de quién se pone. La evaluación puede ser utilizada de forma negativa o inutilizada a través de interpretaciones caprichosas e interesadas (Villalpando y Hernández, 2017). Se puede atribuir la valoración de los protagonistas a su falta de buen criterio o de poca exigencia, se puede achacar la mala opinión de deseos malévolos de perjudicar a los responsables. Esta interpretación que preside el uso de la evaluación no siempre puede estar controlado por el evaluador, sino que, en parte, es responsabilidad del patrocinador o destinatarios de los informes.

Existen diversos modos de hacer inútil o perjudicial una evaluación según dice Santos, incluso una evaluación que ha sido solicitada por los protagonistas de un programa:

1.- Esperar que los resultados de la evaluación sean elogiosos para los responsables de un programa que demanda voluntariamente una evaluación. No es fácil que, sobre todo en los momentos iniciales el informe resulte asimilable en sus vertientes críticas.

2.- Atribuir las informaciones y las explicaciones poco gratas a la subjetividad del evaluador o a la naturaleza cualitativa de la evaluación.

3.- Demandar de la evaluación los juicios de valor que permitan saber a los destinatarios y responsables del programa qué es lo que está bien o lo que está mal hecho. 
4.- Exigir a los evaluadores las orientaciones precisas para la mejora del programa, las instrucciones concretas que provoquen un cambio o las soluciones a los problemas o conflictos existentes.

5.- Poner los resultados de la evaluación al servicio de intereses políticos, económicos, personales, etc., encontrando en los evaluadores una excelente excusa para tomar decisiones sin el compromiso de su justificación auténtica.

6.- Utilizar la evaluación como un arma arrojadiza contra quienes piensan o actúan de forma distinta o contraria, principalmente en el caso de que existan grupos enfrentados en el seno del grupo que desarrolla el programa.

En muchas ocasiones las políticas públicas educativas que pretenden estar encaminadas a lograr una mayor equidad y calidad no representan las demandas y necesidades sociales, no se asumen en una visión integral los verdaderos problemas y por lo tanto son limitados a cubrir cuotas cuantitativas - más escuelas, más profesores, más aulas - cuando en realidad los parámetros son de índole cualitativo (Narro y Moctezuma, 2012). De acuerdo a Aguerrondo (2009) un sistema educativo eficiente no será, entonces, aquél que tenga menos costo por alumno, sino aquél que, optimizando los medios de que dispone sea capaz de brindar educación de calidad a toda la población.

\section{La evaluación educativa como proceso dinámico}

En las sociedades postindustriales se exige cada vez más que la inserción del individuo se produzca equipado con una educación de calidad. Según Delors (1994) es necesario que, en educación, el conocimiento sea el único activo importante, sino que deberá contemplar también la formación en otras capacidades, actitudes y valores en la línea de los expresados por (saber conocer, hacer, convivir y ser).

Ante estos nuevos retos, la evaluación del alumnado, como práctica más extendida para regular la calidad educativa, no puede permanecer anclada en estrategias o enfoques insuficientes o desfasados (Mateo, 2000).

Aprender es un proceso global que abarca a la persona en su conjunto y la lleva a actuar, a 
percibir y a comprender las cosas de distinta manera (Miranda et al., 2017). Existen diferentes enfoques que definen tal concepto: para el conductismo el aprendizaje corresponde a una acción del medio sobre el comportamiento que se traduce en una mejoría estable de los desempeños, no debidos a la maduración. El constructivismo, una posición epistemológica opuesta al conductismo, se asocia al nombre del psicólogo suizo Jean Piaget, quien estudió el desarrollo del pensamiento en el niño para poder esclarecer el funcionamiento cognoscitivo del adulto; el socioconstructivismo de Vygotski, una corriente con un enfoque sociohistórico, también en oposición al conductismo, se distingue del constructivismo de Piaget pues centra el origen del desarrollo del pensamiento en la cultura (Rodríguez, 2010). Desde esta perspectiva, el estudio del aprendizaje, concebido como la apropiación de contenidos de nuevos saberes sociales, no puede llevarse a cabo en forma independiente del estudio de las situaciones de enseñanza que los transmiten.

Podemos afirmar que los procesos de la enseñanza y el aprendizaje están estrechamente relacionados, es decir, son interdependientes. La enseñanza se concibe como un proceso donde interactúan el maestro y el educando, siendo el aprendizaje el objetivo último de este proceso que consiste en dirigir las actividades y utilizar los elementos para facilitar a un individuo, por su propia actividad, aprender. El aprendizaje es la razón de la enseñanza (Horbath y Gracia, 2014).

Por lo anterior, se considera que la evaluación del aprendizaje no sólo valora a los 'aprendientes', sino también al instructor. Según Shepard, citado por Medina (2013) se evalúa la calidad de la relación establecida entre educandos y educadores y los resultados de su interacción: el aprendizaje.

Aceptar el aprendizaje como un proceso dinámico en donde quien aprende debe modificar conscientemente sus propios esquemas cognoscitivos lleva a una visión más amplia de su evaluación (Mejía, 2012). El objeto de estudio es el proceso de aprendizaje como tal, su objetivo es conocer el proceso y resultados del mismo (Foronda y Foronda, 2007). En la capacitación, la evaluación del aprendizaje es un proceso sistemático de investigación, que realizan conjuntamente capacitadores e instructores, para valorar el desarrollo de los conocimientos, habilidades y actitudes propuestos con el fin de retroalimentar el aprendizaje 
de los participantes, la actuación de los instructores y el proceso mismo de capacitación. En este sentido, para Horbath y Gracia (2014) se deben distinguir dos necesidades básicas a las que debe responder la evaluación: la primera, la evaluación como comprensión del proceso de aprendizaje en el grupo en la que el propósito principal es promover la reflexión y participación en el análisis. La segunda, la acreditación, que se relaciona con el requerimiento institucional de certificar si se obtienen los conocimientos que se marcan en los objetivos.

¿De qué manera se deben dinamizar los procesos para el mejoramiento de la evaluación del aprendizaje? Es importante tomar en cuenta que dada la importancia que la educación tiene para el desarrollo social, económico y democrático de un país, es necesario que el sistema educativo informe a la sociedad acerca de lo que los alumnos están aprendiendo (Juárez y Comboni, 2014). Producir y brindar este tipo de información forma parte de los mecanismos de transparencia de la sociedad, al tiempo que contribuye a colocar a la educación como centro de preocupación y como prioridad para la sociedad (Chereguini, 2004).

La información sobre aprendizajes es una herramienta fundamental para la política educativa, en tanto permite identificar y combatir las inequidades dentro del sistema educativo (Márquez, 2012). Algunas décadas atrás, el esfuerzo principal de la política educativa era extender la cobertura. En la medida en que se avanza en la universalización de la cobertura, pasa a ser crucial garantizar que el acceso al sistema educativo se traduzca efectivamente en acceso al conocimiento (Blázquez, 2001).

El énfasis en la evaluación moderna sobre los procesos de enseñanza y de aprendizaje se ha desplazado definitivamente desde la enseñanza hacia los aprendizajes, entendidos en ese sentido amplio a que antes aludíamos (Careaga, 2001). Lo que interesa primordialmente no es lo mucho que el profesor enseña sino lo que el alumno realmente aprende.

No se puede gestionar eficazmente la evaluación, si se pretende atender a sus nuevas responsabilidades, desde la acción individual del profesor o concibiendo las asignaturas como entidades aisladas (Mateo, 2000). La gestión de la evaluación moderna exige el trabajo mancomunado de los profesores y sitúa el currículum como referente básico del 
diseño educativo y evaluativo. En consecuencia, la nueva gestión no puede fundamentarse en la recogida de información centrada fundamentalmente en la instrumentación clásica. Las pruebas de ejecución basadas en los planteamientos alternativos de la evaluación, deberán complementar o sustituir los modelos más tradicionales, si se quieren articular estrategias que capturen información realmente sustantiva (Fandos, 2003).

En los procesos de evaluación, las pruebas de referencia de criterios deben constituirse en la alternativa más común a aplicar en la nueva instrumentación. Hay que incorporar a otros agentes, como puedan ser los propios alumnos, en las responsabilidades evaluativas (Calderón, 2015). Sólo desde procesos de autoevaluación es posible desarrollar a fondo una verdadera autonomía en los aprendizajes. Corresponsabilizar en alguna medida a los alumnos en los procesos evaluativos constituirá un importante reto en el futuro.

La lógica de la evaluación, por sí sola, no garantiza la asunción de calidad (Mateo, 2000). La innovación en la evaluación de los aprendizajes supone la aplicación coordinada de un conjunto importante de distintas estrategias. Una evaluación auténtica es por naturaleza multidireccional, directa (Calderón, 2015). Su uso ha de ser intensivo de forma que produzca retroalimentación continua y rápida.

Los criterios evaluativos no deben ser ni ocultos ni misteriosos sino transparentes (Pérez, 2008). La actitud de profesores y alumnos no puede ser pasiva, sino que todos deben estar activados y perfectamente ensamblados en un proyecto que lo perciben como común. La evaluación desde esta perspectiva cultural implica revaluación y autoevaluación hasta alcanzar los logros de aprendizaje previstos (Mateo, 2000).

Debemos de ser enfáticos en que los objetivos de la nueva evaluación no se sitúan en el control sino en la mejora, en la optimización de la capacidad de aprendizaje de los alumnos, de enseñanza de los profesores y de creación de entornos favorecedores de ambos por parte de la institución (Navarro et al., 2017). Es obvio que las dificultades desde esta perspectiva surgirán de la inconformidad al cambio del pensamiento tanto de profesores como de alumnos. Nuestra sociedad está acostumbrada a otras metáforas, y modificar hábitos sobre prejuicios tan enquistados en las rutinas supondrá indudablemente un 
importante esfuerzo (Mateo, 2000).

Muchos profesores, en todos los niveles educativos, admiten la dificultad de contactar con sus alumnos llegándolos a percibir como difíciles de conocer y de predecir. Una actitud frecuentemente expresada por parte del profesorado ante el alumnado es la de desconcierto, que evidentemente constituirá uno de los elementos con los que habrá que contar en la construcción de nuevas formas evaluativas (Ahumada, 2005).

Las características generales aquí señaladas cuestionan la posibilidad de establecer modelos únicos de evaluación, de aquí la importancia de introducir la necesidad de que la evaluación se sustente sobre una multiplicidad de fuentes y evidencias (Cárdenas, 2015). Debe admitir diversos puntos de vista, compartir juicios y ha de ser permanentemente redefinible. En general es fundamental el aceptar como su carácter más propio la pluralidad (Martín, 2010).

La aseveración de Popham (1999) en cuanto a que los educadores viven una presión implacable para demostrar su eficacia es puesta de relieve a la par de la inconformidad magisterial por ser evaluados con instrumentos poco claro y pertinente. Está claro que en la reforma educativa la evaluación es un eje estratégico, a partir del cual se espera obtener información valiosa para realizar tareas de rediseño institucional y organizacional (al menos así está plasmado en la ley), en la que la toma de decisiones esté fundamentada en información que evidencie los errores a corregir. Por ello, la idoneidad de los instrumentos de evaluación es fundamental, pues son mediante estos como se obtendrá la información base para la toma de decisiones (Gallardo et al., 2012).

A decir de Medina (2013) históricamente el desarrollo en la psicometría se ha realizado en contextos de las pruebas a gran escala, por expertos en estadística y rara vez por docentes o expertos de una disciplina. Posteriormente se han "transferido" dichos conocimientos al contexto de la evaluación del salón de clases. En otras palabras, en lugar de desarrollar una teoría o pensamiento propios y emergidos del salón de clase, se procedió a "pedir prestada" la teoría formulada para contextos de la evaluación de gran escala o masiva. Por esta razón resulta peligroso interpretar los resultados de las pruebas estandarizadas a la luz de la 
calidad educativa de un plantel escolar (Hernández et al., 2018). Asimismo, Popham (1999) señala que es engañoso establecer la eficacia de un maestro con el alto puntaje que hubieran arrojado los alumnos, puesto que las pruebas contabilizan e interpretan aptitudes, mas no los avances en la enseñanza aprendizaje, aunado a la escala tan pequeña en la aplicación de la prueba.

Es pertinente considerar los contextos educativos al momento de echar a andar las acciones para evaluar, es decir, que se tome en cuenta a todos los elementos adecuados de la realidad sociocultural. De acuerdo con Medina (2013) existen diferencias sobre las nuevas corrientes psicopedagógicas. Autores como Shepard afirman que la disonancia actual emerge por la incompatibilidad entre las viejas ideas de los tests y las ideas transformadas de la enseñanza. Lo que adquiere especial relevancia si se considera la premisa de De la Orden (2011) que sugiere que el enfoque de la educación determina el qué evaluar (su objeto), el cómo evaluar (su método) y el cuándo evaluar (su planeación). En resumen, se trata de buscar equilibrio, estabilidad y consistencia a lo que se evalúa. Nuevas medidas de evaluación para las nuevas formas del proceso educativo.

Es importante distinguir evaluaciones que buscan llegar a juicios sobre individuos (sean estos alumnos, docentes, centros escolares $u$ otros), en contraste con las que buscan alcanzar conclusiones sobre un sistema educativo en conjunto (Murphy, 2002). En el caso de evaluaciones de aprendizaje, unas sirven para dar resultados de cada alumno, sea para decidir si pasa al grado siguiente o debe repetir el mismo curso; sea para tener elementos para ajustar sus esfuerzos para mejorar el aprendizaje ulterior. Otras evaluaciones, en cambio, sirven para informar sobre la situación promedio del aprendizaje de los alumnos de cierto nivel, y para sustentar decisiones de política que afecten al conjunto del sistema educativo (Martínez, 2013). En el primer caso, es necesario aplicar las pruebas a todos los alumnos (censo), mientras que en el segundo basta con hacerlo a una muestra representativa, lo que permite usar instrumentos que midan aspectos más complejos.

Para evaluar cada una de las dimensiones de la calidad de un sistema educativo se necesitan distintos acercamientos. Las pruebas de aprendizaje sólo sirven para evaluar ese aspecto, pero para otros se deberán manejar indicadores basados en estadísticas. Se 
requerirán estudios especiales, a veces de gran complejidad, como los que son necesarios para la evaluación de docentes (Martínez, 2013). Los riesgos de malos usos de este tipo de evaluaciones, sin embargo, están presentes por el desconocimiento de las cuestiones técnicas por parte de muchos tomadores de decisiones, junto a la presencia de intereses ajenos a lo que tendría que ver estrictamente con la mejora de la calidad educativa en el sentido más completo de la expresión, incluyendo las dimensiones de relevancia y equidad (Guzmán, 2018).

\section{El reto de la comunicación y la información}

Sin contar las pruebas de ingreso a la universidad $u$ otros programas educativos, los resultados de las pruebas de aprendizaje en gran escala que se aplicaban en los países de la región latinoamericana no se usaban, en general, para tomar decisiones que afectaran a personas o instituciones en lo individual. Las pruebas no se utilizaban para decidir sobre la aprobación o reprobación de los alumnos, ni tampoco para asignar estímulos o tomar medidas correctivas que afectaran a maestros o escuelas individuales (Márquez, 2014). Por ello las pruebas podían definirse como de bajo impacto. En algunos casos su impacto era de hecho nulo, por la ausencia de difusión de los resultados (Martínez, 2016).

La proliferación de las pruebas en años más recientes, en especial ya en el siglo XXI, ha ido acompañada por un creciente interés porque sus resultados sean utilizados para sustentar decisiones de las que se espera se deriven mejoras importantes de la calidad educativa. Esta tendencia se relaciona con la noción de rendición de cuentas y cobra sentido en el contexto de otras corrientes más amplias. Por una parte, la búsqueda de mayor transparencia en el manejo de los asuntos públicos. Por otra parte, aunque con matices importantes, cierta desconfianza respecto de la educación pública y, en general, respecto a la gestión pública de los servicios (Martínez, 2009). A lo anterior debe añadirse la escasa cultura que hay en las sociedades latinoamericanas en cuanto a evaluación educativa, y no sólo entre el público en general, sino también entre maestros y autoridades educativas, e incluso entre investigadores y especialistas. La combinación de los elementos anteriores está llevando, en varios casos, a que se esperen resultados casi milagrosos de mejora de las escuelas gracias a la aplicación de pruebas de aprendizaje en gran escala, sin tener en cuenta sus reales alcances y sus limitaciones (Martínez, 2016). 
La evaluación más importante, una que incluya todos los aspectos del currículum y los niveles cognitivos más complejos, teniendo en cuenta las circunstancias de cada alumno, sólo puede hacerla un buen maestro, en el contexto del aula, con acercamientos mucho más ricos que los que pueden emplearse en gran escala (García et al., 2011). Las pruebas de este tipo deben ser vistas como un complemento del trabajo de los maestros, y no como un sustituto de él. Es fundamental un cambio de perspectiva, que deje de ver a la evaluación como amenaza externa, para pasar a considerarla una excelente oportunidad de aprendizaje.

Dos riesgos están presentes, la falta de continuidad que traen los cambios políticos cuyo efecto destructor se ha constatado, y el peligro de los efectos perversos para la calidad educativa que traen consigo prácticas derivadas de una comprensión inadecuada de los alcances y limitaciones de las evaluaciones en gran escala (Calderón, 2015). La reciente tendencia a hacer evaluaciones censales puede ser positiva, pero también un avance en dirección negativa. En contraste con lo que pasaba hace una década, cuando las evaluaciones eran pocas y sus resultados casi no se difundían, hoy las aplicaciones proliferan y comienzan a cansar a las escuelas; los resultados se debaten, a veces con escaso conocimiento de su complejidad. Para que las perspectivas favorables puedan concretarse será necesario que una visión objetiva de las posibilidades de la evaluación prevalezca sobre las visiones superficiales (Taboadam et al., 2020).

Los resultados de las mediciones, asumidos por los medios de comunicación con gran repercusión, tienen un enorme impacto político que no se contradice con la relativa solidez técnica que tienen algunos de los instrumentos utilizados, ni con la capacidad de dichos medios para interpretar correctamente las informaciones. El efecto desmoralizador de la difusión de los resultados supera la capacidad movilizadora para mejorarlos (Álvarez, 2001). Es importante que aprendamos a distinguir claramente la diferencia que existe entre satisfacer demandas y satisfacer necesidades. La capacidad de demanda está desigualmente distribuida y si las políticas educativas se limitan a satisfacer las demandas, se tiende a dejar a cada uno en el lugar que le permite su capacidad de demandar (Tedesco, 2016). Transformar la necesidad en una demanda es un proceso complejo, no automático. 
Por ello, es necesario pensar en un futuro deseable de la difusión de los resultados evaluadores, en la cual los datos lleguen de manera sistemática y pertinente a todos los actores involucrados. Esto implica que no sólo se deberán difundir de la manera más amplia posible, sino también ofrecer interpretaciones que tomen en cuenta los diferentes contextos (por ejemplo, el cultural o el económico) en los cuales ocurren los diversos resultados, sólo así se dará lugar a una evaluación relevante para las distintas necesidades y demandas en cada nivel del sistema. Será imprescindible, por lo tanto, fortalecer los mecanismos existentes de capacitación para la interpretación y el uso de la información, así como establecer otros nuevos (Álvarez, 2001).

La difusión de resultados es una dimensión fundamental de la evaluación, dado que confiere sentido a los esfuerzos realizados. Una difusión adecuada a las exigencias y necesidades de los diferentes actores educativos, es indispensable para el diseño e implementación de políticas educativas eficaces, así como para la rendición de cuentas frente a la sociedad. No obstante, no toda forma de difusión es igualmente deseable (Santos, 2001). No es suficiente con garantizar el acceso a un gran volumen de información, sino que deben ofrecerse las herramientas adecuadas para su interpretación, así como evitar usos inapropiados (Fandos, 2003). Esto implica tener en cuenta las características de los destinatarios y potenciales usuarios de la información, cuyas atribuciones, necesidades y capacidades son diversas.

Debemos de entender que la evaluación educativa no es un fin en sí misma, sino un medio que cobra sentido en la medida en que contribuye a que la educación mejore. El uso de las evaluaciones por parte de los diferentes actores educativos aún no muestra la organización o el nivel de aprovechamiento que serían deseables (INEE, 2019). Esto se debe a que la cultura de la evaluación en México todavía necesita afianzarse y normarse, dado que la difusión en sí misma es un fenómeno relativamente nuevo.

La evaluación es esencial para la calidad educativa, ya que arroja distintas clases de información que permiten tomar decisiones mejor informadas y entender procesos de enseñanza-aprendizaje que no son tan claros sin su aplicación (Jordán, et al. 2017). El uso pedagógico de los resultados orienta el trabajo de las instituciones, los docentes, los estudiantes y los padres de familia (Valdés y Urías, 2011). De ahí la importancia de verla 
como una herramienta para potenciar los aprendizajes y los procesos que ocurren en el aula, dentro del ciclo de calidad que busca fortalecer las instituciones educativas y conjuga estándares básicos de competencia, procesos de evaluación y diseño e implementación de planes de mejoramiento institucional.

Las autoridades educativas han empezado a percibir la importancia de la evaluación como herramienta imprescindible en la toma de decisiones (INEE, 2006). Es muy importante lograr avances en este nivel. Deben ser las propias entidades quienes fomenten el uso de la información por parte de los sectores intermedios y de las escuelas. Para lograr que los resultados de las evaluaciones lleguen debidamente contextualizados, deberá privilegiarse su difusión en la agenda educativa de las entidades (INEE, 2019). A nivel de las escuelas, la difusión de los resultados es considerablemente heterogénea, ya que depende de múltiples factores, desde geográficos hasta políticos.

La información que proporciona la evaluación sirve para que los maestros dispongan de información relevante con el fin de analizar críticamente su propia intervención educativa y tomar decisiones al respecto (Anglas, 2007). Sin embargo, la realidad educativa en América Latina, está muy distante de lograr dicho objetivo, sobre todo en el renglón del uso y difusión de esa información valiosa. Es importante subrayar que, en el momento de la comunicación de datos, de informar a la sociedad sobre los resultados de la evaluación, se debe de hacer como un acto de reflexión, sin el cual, toda la actividad se convertiría en un acto vacío de estadística sin proyección.

\section{CONCLUSIONES}

Si partimos de la premisa máxima de que la educación es un derecho humano fundamental para una vida digna, en la que se desarrollen todas las potencialidades y capacidades del individuo para enfrentar los retos cotidianos, no podemos dejar de lado que la evaluación de los procesos educativos es tan importante para rescatar y poner de relieve todos los elementos clave para el mejoramiento de la cobertura, acceso y permanencia a una educación de calidad.

Creemos que una mayor participación de la sociedad civil hará posible que la evaluación 
educativa posea profundidad y alcances de largo plazo, por esta razón estamos de acuerdo con el hecho de que se identifique la evaluación con la medición, para el caso de la evaluación que realizan los docentes en el aula; preocupa también el énfasis en ésta como un proceso relacionado principalmente con la planeación del aprendizaje, cuando, desde la perspectiva de la evaluación formativa, es continua y forma parte del proceso mismo de realización. Siendo así, la evaluación educativa se reflejará con claridad en los procesos de aprendizaje y un profundo respeto de los contextos de personalización y colaboración.

La difusión de resultados es la dimensión fundamental de una buena evaluación. De hecho, podría afirmarse que constituye el sentido último de todo el sistema. Es importante que los resultados de evaluación para su mejor uso, sean interpretados de manera profesional y ética (Martínez y Blanco, 2010) con la intención de no ser amenazados por otros que tengan tintes de ideologías burocráticas o grupo social con intereses ajenos a la educación.

Suena absurdo, pero es la realidad; tanto la estadística como la psicología han aportado a la educación los instrumentos y teorías que no necesariamente surgieron en y para el aula. Insistimos que es el momento para reflexionar y poner en acción medidas acordes a la nueva realidad educativa. Iniciar con la discusión de los propósitos, validez y confiabilidad de las herramientas evaluatorias. Se necesita que el sistema educativo formule, diseñe e implemente sus propias pruebas, que sea el docente junto con los padres de familia quienes actúen en primera línea. La evaluación educativa la han de realizar los responsables directos. La educación de los hijos de una nación es un asunto demasiado importante, pedagógicamente útil y socialmente significativa. En resumen, se trata de buscar equilibrio, estabilidad y consistencia a lo que se evalúa; nuevas medidas de evaluación para las nuevas formas del proceso educativo.

\section{REFERENCIAS BIBLIOGRÁFICAS}

Aguerrondo, Inés (2009). La calidad de la educación: Ejes para su definición y evaluación.

Recuperado

de:

http://formaciondocente.com.mx/Bibliotecadigital/18_TemasEducacion/La\%20Cali dad\%20de\%20la\%20Educacion.pdf

Ahumada A., Pedro (2005). La evaluación auténtica: un sistema para la obtención de evidencias y vivencias de los aprendizajes. Perspectiva Educacional, Formación de 
Profesores, (45), 11-24 ISSN: 0716-0488. Recuperado de: https://www.redalyc.org/articulo.oa?id=3333/333329100002

Anglas, Miguel (2007). Evaluación: proceso para la toma de decisiones. Recuperado de: http://blog.pucp.edu.pe/blog/anglas/2007/10/10/evaluaci-n-proceso-para-la-tomade-decisiones/

Álvarez Méndez, Juan Manuel (2001). Evaluar para conocer, examinar para excluir. Recuperado de: http://www.fceia.unr.edu.ar/geii/maestria/2013/10masCelman/\%C3\%81/varez\%20 M\%C3\%A9ndez-

Evaluar\%20para\%20conocer\%20examinar\%20para\%20excluir006.pdf

Blázquez Entonado, Florentino (2001). La sociedad de la información y la comunicación, reflexiones desde la educación. Recuperado de: http://www.ub.edu/prometheus21/articulos/obsciberprome/blanquez.pdf

Calderón, David (2015). Evaluación, validez y relevancia. Revista Mexicana de Investigación educativa.

Recuperado

de:

http://www.mexicanosprimero.org/index.php/educacion-en-mexico/nuestraopinion/item/evaluacion-validez-y-relevancia

Cárdenas Saldaña, Norma Eugenia (2015). Tesis, Análisis crítico del sistema de evaluación del desempeño profesional docente chileno: un estudio en la comunidad educativa de la ciudad de Osorno, Chile. Universitat Autónoma de Barcelona. Recuperado de: https://ddd.uab.cat/pub/tesis/2016/hdl_10803_384556/necs1de1.pdf

Careaga, Adriana (2001). La evaluación como herramienta de transformación de la práctica docente. Educere, 5 (15), 345-352. ISSN: 1316-4910. Recuperado de: https://www.redalyc.org/articulo.oa?id=356/35651519

Casanova, María Antonieta (2002). Ámbitos de la educación. Instituto Hidalguense de Educación, Universidad Pedagógica Nacional-Hidalgo. Recuperado de: http://upnmorelos.edu.mx/assets/evaluacion-educativa.pdf

Chereguini, Estefanía (2004). Sociedad de la Información al servicio de los pueblos. La Sociedad de la Información en el siglo XXI: un requisito para el desarrollo. Buenas 
prácticas y lecciones aprendidas. Recuperado de: https://www.itu.int/net/wsis/stocktaking/docs/activities/1103547250/sociedadinformacion-sigloxxi-es.pdf

Delors, Jacques (1994). Los cuatro pilares de la educación. La educación encierra un tesoro. México: El Correo de la UNESCO, pp. 91-103. Recuperado de: https://www.uv.mx/dgdaie/files/2012/11/CPP-DC-Delors-Los-cuatro-pilares.pdf

De la Orden Hoz, Arturo (2011). Reflexiones en torno a las competencias como objeto de evaluación en el ámbito educativo. Revista Electrónica de Investigación Educativa. Recuperado de: https://redie.uabc.mx/redie/article/view/278

Fandos Garrido, Manuel (2003). Tesis doctoral: Formación basada en las Tecnologías de la Información y Comunicación: Análisis didáctico del proceso de enseñanzaaprendizaje.

Recuperado de: https://www.tdx.cat/bitstream/handle/10803/8909/Etesis_1.pdf

Faustino Zacarías, Oscar Hugo (2016). Pertinencia de los instrumentos para evaluar el desempeño docente. Recuperado de: http://www.educacionfutura.org/pertinenciade-los-instrumentos-para-evaluar-el-desempeno-docente/

Fernández Ludeña, Alejandro (2008). La educación para el trabajo en un mundo globalizado. Una mejor educación para una mejor sociedad: Propuestas para el diálogo y la transformación educativa en América Latina y el Caribe. Recuperado de: https://www.oei.es/historico/pdf2/mejor_educ_mejor_sociedad.pdf

Foronda Torrico, José María y Foronda Zubieta, Claudia Lorena (2007). La evaluación en el proceso de aprendizaje. perspectivas. ISSN: 1994-3733. Disponible en: https://www.redalyc.org/articulo.oa?id=4259/425942453003

Gallardo Córdova, Katherina Edith, Gil Rendón, María Eugenia, Contreras Durán, Baltazar, García Hernández, Evelyn, Lázaro Hernández, Roger Alberto, \& Ocaña Jiménez, Lilibeth. (2012). Toma de decisiones para la evaluación formativa: el proceso de planeación y determinación de sus mecanismos. Sinéctica, (39), 01-19. Recuperado de: 
http://www.scielo.org.mx/scielo.php?script=sci_arttext\&pid=S1665-

109X2012000200011\&lng=es\&tlng=es.

García Medina, Adán M.; Aguilera García, María A-; Pérez Martínez, María G.; Muñóz Abundez, Gustavo (2011). Evaluación de los aprendizajes en el aula Opiniones y prácticas de docentes de primaria en México. Instituto Nacional para la Evaluación de la Educación Recuperado de: http://uprid2.up.ac.pa:8080/xmlui/handle/123456789/2098?show=full

Guzmán Marín, Francisco (2018). La experiencia de la evaluación docente en México: Análisis crítico de la imposición del servicio profesional docente. Red Iberoamericana de Investigación sobre Cambio y Eficacia Escolar (RINACE). Recuperado de: http://hdl.handle.net/10486/681784

Hernández Madrigal, Mónica, Ramírez Flores, Élfego, y Gamboa Cerda, Silvia. (2018). La implementación de una evaluación estandarizada en una institución de educación superior. Innovación educativa (México, DF), 18(76), 149-170. Recuperado de: http://www.scielo.org.mx/scielo.php?script=sci_arttext\&pid=S166526732018000100149\&lng=es\&tlng=es.

Horbath, Jorge E. Gracia, Ma. Amalia (2014). La evaluación educativa en México. Revista de Relaciones Internacionales, Estrategia y Seguridad. Recuperado de: http://www.scielo.org.co/scielo.php?script=sci_arttext\&pid=S190930632014000100004

INEE (2006). Políticas y sistemas de evaluación educativa en México: Avances, logros y desafíos. Informe para la Reunión Ministerial del Grupo E-9 (UNESCO). Recuperado de: https://www.inee.edu.mx/wp-content/uploads/2019/01/P1D206.pdf

INEE-SEP (2006). Sistema de Indicadores Educativos de los Estados Unidos Mexicanos. Conjunto básico para el ciclo 2004-2005. México. Recuperado de: https://historico.mejoredu.gob.mx/wp-content/uploads/2018/12/P1C308.pdf

INEE (2019). La difusión de los resultados de las evaluaciones. Los Temas de la Evaluación. colección de folletos ISSN 1665-9465 Recuperado de: https://www.inee.edu.mx/wp-content/uploads/2019/01/P1C610.pdf 
Jiménez, Jiménez Bonifacio (2002). La evaluación: su conceptualización. Instituto Hidalguense de Educación, Universidad Pedagógica Nacional-Hidalgo. Recuperado de: http://upnmorelos.edu.mx/assets/evaluacion-educativa.pdf

Jordán, Y., Morán, B. y Camacho, T. (2017). La evaluación de los aprendizajes y su influencia en la calidad del proceso de enseñanza aprendizaje en el contexto universitario. Opuntia Brava 9 (1). Recuperado de http://www. opuntiabrava.edu.cu9

Juárez Núñez, José Manuel, y Comboni Salinas, Sonia. (2014). La influencia de la Organización para la Cooperación y el Desarrollo Económicos en la Reforma Educativa. Argumentos (México, D.F.), 27(74), 153-189. Recuperado de: http://www.scielo.org.mx/scielo.php?script=sci_arttext\&pid=S0187$57952014000100007 \&$ Ing=es\&tlng=es.

Lafourcade, D. Pedro. (2002). La evaluación en el proceso educacional. Instituto Hidalguense de Educación, Universidad Pedagógica Nacional-Hidalgo. Recuperado de: http://upnmorelos.edu.mx/assets/evaluacion-educativa.pdf

Márquez Jiménez, Alejandro. (2012). El financiamiento de la educación en México: Problemas y alternativas. Perfiles educativos, 34(spe), 107-117. Recuperado de: http://www.scielo.org.mx/scielo.php?script=sci_arttext\&pid=S0185$26982012000500010 \&$ lng=es\&tlng=es.

Márquez Jiménez, Alejandro. (2014). Las pruebas estandarizadas en entredicho. Perfiles educativos, 36(144), 3-9. Recuperado de: http://www.scielo.org.mx/scielo.php?script=sci_arttext\&pid=S0185$26982014000200001 \&$ lng=es\&tlng=es.

Martín, Elena (2010). ¿En qué medida la evaluación está contribuyendo a la mejora de la calidad de la educación? En: Presente y futuro de la educación Iberoamericana. Universidad Autónoma de Madrid. Recuperado de: https://dialnet.unirioja.es/servlet/articulo?codigo=7011204

Martínez Rizo, Felipe. (2009). Consideraciones sobre la evaluación educativa. Recuperado de: http://www.oei.es/historico/metas2021/expertos20.htm 
Martínez Rizo, Felipe. (2013). El futuro de la evaluación educativa. Sinéctica, (40), 01-11.

Recuperado

de:

http://www.scielo.org.mx/scielo.php?script=sci_arttext\&pid=S1665-

109X2013000100006\&lng=es\&tlng=es.

Martínez Rizo, Felipe (2016). Impacto de las pruebas en gran escala en contextos de débil tradición técnica: Experiencia de México y el Grupo Iberoamericano de PISA. RELIEVE, 22 (1), art. MO. Recuperado de: http://dx.doi.org/10.7203/relieve.22.1.8244

Martínez Rizo, Felipe y Blanco, Emilio (2010). La evaluación educativa en México: experiencias, avances y desafíos. Recuperado de: http://docplayer.es/12124546La-evaluacion-educativa-en-mexico-experiencias-avances-y-desafios.html

Mateo, J.A. (2000). La evaluación educativa, su práctica y otras metáforas. ICE, Universidad de Barcelona. Recuperado de: https://web.ua.es/es/ice/documentos/recursos/materiales/la-evaluacioneducativa.pdf

Medina Gual, Luis (2013). La evaluación en el aula: reflexiones sobre sus propósitos, validez y confiabilidad. Revista Electrónica de Investigación Educativa. Recuperado de: https://redie.uabc.mx/redie/article/view/444/608

Mejía Pérez, Omar (2012). De la evaluación tradicional a una nueva evaluación basada en competencias. Revista Electrónica Educare Vol. 16, № 1, [27-46], ISSN: 1409-4258 Recuperado de: https://dialnet.unirioja.es/descarga/articulo/3975651.pdf

Miranda López, Francisco; Islas Dossetti, Juana y Fuentes Bonifacio, Aracely (2017). E Sistema Nacional de Evaluación Educativa en México: un análisis en perspectiva comparada. Recuperado de: http://www.comie.org.mx/congreso/memoriaelectronica/v14/doc/2095.pdf

Murphy, D. (2002). El desarrollo de una cultura de la evaluación. Íkala. 7 Revista de lenguaje y cultura. Universidad de Antioquia, Colombia. (1), pp. 75 - 85 Recuperado de: https://www.redalyc.org/pdf/2550/255026059006.pdf 
Narro Robles, José; Moctezuma Navarro, David (2012). Hacia una reforma del Sistema Educativo Nacional. Recuperado de: http://www.planeducativonacional.unam.mx/PDF/completo.pdf

Navarro Mosquera, Narda Gisela, Falconí Asanza, Aldo Vinicio, \& Espinoza Cordero, Jessica. (2017). El mejoramiento del proceso de evaluación de los estudiantes de la educación básica. Revista Universidad y Sociedad, 9(4), 58-69. Recuperado de: http://scielo.sld.cu/scielo.php?script=sci_arttext\&pid=S221836202017000400008\&lng=es\&tlng=es.

Pérez Morales, Juana Idania (2008). La evaluación como instrumento de mejora de la calidad del aprendizaje. Propuesta de intervención psicopedagógica para el aprendizaje del idioma inglés. Universitat de Girona. Recuperado de: https://www.tdx.cat/bitstream/handle/10803/8004/tjipm.pdf

Popham, W. J. (1999). ¿Por qué las pruebas estandarizadas no miden la Calidad Educativa? Tomado y traducido de Educational Leadership, volumen 56, número 6, marzo de 1999, con autorización de ASCD (editores).

Red, Revista de Evaluación para docentes y directivos. Número 08 (septiembre-diciembre 2017). Instituto Nacional de Evaluación Educativa http://www.inee.edu.mx/Red2017/Red08/Red08flip.html

Rodríguez Arocho, Wanda (2010). El concepto de calidad educativa: una mirada crítica desde el enfoque histórico cultural Revista Electrónica Actualidades Investigativas en Educación, vol. 10, núm. 1, enero-abril, 2010, pp. 1-28 Universidad de Costa Rica. San Pedro de Montes de Oca, Costa Rica. Recuperado de: http://www.redalyc.org/pdf/447/44713068015.pdf

Ruíz Ruíz, José María (1996). Cómo hacer una evaluación de centros educativos. Madrid, editorial Narcea.

Santos, Guerra Miguel Ángel (2001). Sentido y finalidad de la evaluación de la universidad. Revista, Perspectiva Educacional. Recuperado de: www.uaa.mx/direcciones/dgdp/defaa/descargas/evaluaciondelauniversidad.pdf 
Santos, Guerra Miguel Ángel (2002). La evaluación: un proceso de diálogo, comprensión y mejora. Instituto Hidalguense de Educación, Universidad Pedagógica NacionalHidalgo. Recuperado de: http://upnmorelos.edu.mx/assets/evaluacioneducativa.pdf

Taboada Galas, Cecilia; Gutiérrez Cortés, María Teresa y Hamilton Vélez, Eva María (2020). Uso de Resultados de las pruebas de aprendizaje en el diseño de las políticas educativas en América Latina. IIPE Unesco. Recuperado de: https://www.buenosaires.iiep.unesco.org/sites/default/files/archivos/Estado\%20del \%20arte\%20-\%20Cecilia\%20Galas_1.pdf

Tedesco, Juan Carlos (2016). Diez notas sobre los sistemas de evaluación de los aprendizajes. Oficina Internacional de Educación. UNESCO. Recuperado de: http://www.ibe.unesco.org/es/blog/diez-notas-sobre-los-sistemas-deevaluaci\%C3\%B3n-de-los-aprendizajes

Valdés Cuervo, Ángel Alberto, y Urías Murrieta, Maricela. (2011). Creencias de padres y madres acerca de la participación en la educación de sus hijos. Perfiles educativos, 33(134), 99-114.

Recuperado de:

http://www.scielo.org.mx/scielo.php?script=sci_arttext\&pid=S018526982011000400007\&lng=es\&tlng=es.

Villalpando Sifuentes, Celia Gabriela, y Hernández Pérez, Claudio. (2017). Evaluación para la permanencia: una visión desde la experiencia docente. IE Revista de investigación educativa de la REDIECH, 8(14), 31-43. Recuperado de: http://www.scielo.org.mx/scielo.php?script=sci_arttext\&pid=S2448$85502017000100031 \&$ lng=es\&tlng=es.

Zorrilla, M. (2002). Diez años después del Acuerdo Nacional para la Modernización de la Educación Básica en México: Retos, tensiones y perspectivas. Revista Electrónica de Investigación Educativa, 4. Recuperado de: http://redie.uabc.mx/vol4no2/contenido-zorrilla.html 